\title{
Acesso aos serviços de saúde bucal de crianças e adolescentes com deficiência em um município de grande porte do Nordeste do Brasil
}

Access to oral health services of children and adolescents with disabilities in a large municipality in Northeast Brazil

\author{
Acceso a los servicios de salud bucal de niños y adolescentes con discapacidad en un gran municipio \\ del Noreste de Brasil
}

Recebido: 16/04/2021 | Revisado: 23/04/2021 | Aceito: 27/04/2021 | Publicado: 12/05/2021

\author{
Andreza Cristina de Lima Targino Massoni \\ ORCID: https://orcid.org/0000-0002-3332-8315 \\ Universidade Estadual da Paraíba, Brasil \\ E-mail: andrezatargino@gmail.com \\ Murilo Áquila de Oliveira Viana \\ ORCID: https://orcid.org/0000-0002-9058-3611 \\ Universidade Estadual da Paraíba, Brasil \\ E-mail: murilloodonto@hotmail.com \\ Érika Porto \\ ORCID: https://orcid.org/0000-0002-0287-6933 \\ Universidade Estadual da Paraíba, Brasil \\ E-mail: erikaporto1@gmail.com \\ Álisson Thiago Lima \\ ORCID: https://orcid.org/0000-0002-7928-8865 \\ Universidade Estadual da Paraíba, Brasil \\ E-mail: legionalisson@gmail.com \\ Pablo Jardel de Oliveira Santos \\ ORCID: https://orcid.org/0000-0002-8736-2498 \\ Universidade Estadual da Paraíba, Brasil \\ pablojjardel@gmail.com \\ Lydiane dos Santos Dantas \\ ORCID: https://orcid.org/0000-0003-1125-6872 \\ Universidade Estadual da Paraíba, Brasil \\ E-mail: lydiane.dantaas@gmail.com
}

\begin{abstract}
Resumo
Objetivo: Identificar o acesso aos serviços de saúde bucal e as dificuldades no atendimento odontológico de crianças e adolescentes com deficiência, institucionalizados, em Campina Grande, PB. Métodos: Realizou-se uma pesquisa observacional, descritiva, quantitativa, do tipo transversal com cuidadores de crianças e adolescentes com deficiência, que frequentavam a APAE-CG. Os dados foram analisados através de técnicas de estatística descritiva por distribuições absolutas e percentuais. Resultados: Observou-se que 64,6\% das crianças e adolescentes já haviam sido levadas ao Dentista, sendo o serviço mais buscado, a Estratégia Saúde da Família (ESF) (58,0\%). 67,9\% afirmaram não ter encontrado dificuldades de acesso. Entre os que relataram ter dificuldade, a queixa principal referiu-se à recusa do profissional da ESF em atender pacientes com deficiência (52,9\%). Conclusão: A maioria das crianças e adolescentes, usuários da APAE-CG, já havia sido levada ao Dentista. A queixa principal quando do não atendimento, referiu-se à recusa dos profissionais da ESF.

Palavras-chave: Pessoas com deficiência; Assistência odontológica para pessoas com deficiências; Acesso aos serviços de saúde; Saúde bucal.

Abstract

Objective: To identify access to oral health services and difficulties in dental care for children and adolescents with disabilities, institutionalized, in Campina Grande, PB. Methods: An observational, descriptive, quantitative, crosssectional study was conducted with caregivers of children and adolescents with disabilities, who attended the APAECG. The data were analyzed using techniques of descriptive statistics by absolute and percentage distributions. Results: It was observed that $64.6 \%$ of children and adolescents had already been taken to the Dentist, with the most sought service being the Family Health Strategy (FHS) (58.0\%). 67.9\% stated that they did not find access difficulties. Among those who reported having difficulty, the main complaint referred to the FHS professional's refusal to treat patients with disabilities (52.9\%). Conclusion: Most children and adolescents, users of APAE-CG, had already been taken to the Dentist. The main complaint regarding non-attendance, referred to the refusal of the ESF professionals.
\end{abstract}


Keywords: Disabled persons; Dental care for disabled; Health services accessibility; Oral health.

\section{Resumen}

Objetivo: Identificar el acceso a los servicios de salud bucal y las dificultades en la atención odontológica de niños y adolescentes con discapacidad, institucionalizados, en Campina Grande, PB. Métodos: se realizó un estudio observacional, descriptivo, cuantitativo, transversal con cuidadores de niños y adolescentes con discapacidad, que asistieron a la APAE-CG. Los datos se analizaron mediante técnicas de estadística descriptiva mediante distribuciones absolutas y porcentuales. Resultados: Se observó que el 64,6\% de los niños y adolescentes ya habían sido llevados al Odontólogo, siendo el servicio más buscado la Estrategia de Salud de la Familia (ESF) (58,0\%). El 67,9\% afirmó no encontrar dificultades de acceso. Entre los que refirieron tener dificultades, la principal queja se refirió a la negativa del profesional de la ESF a atender a pacientes con discapacidad (52,9\%). Conclusión: La mayoría de los niños y adolescentes usuarios de APAE-CG ya habían sido llevados al Odontólogo. La principal queja cuando no fue contestada, se refería a la negativa de los profesionales de la ESF.

Palabras clave: Personas com discapacidad; Atención dental para personas con discapacidades; Accesibilidad a los servicios de salud; Salud bucal.

\section{Introduçãa}

O conceito de deficiência é reconhecido como algo que está em evolução. Destaca-se a necessidade de se observar a interação entre pessoas com deficiência e as possíveis barreiras geradas pelo ambiente em que estas estão inseridas (Câmara, 2009); e a partir daí refletir sobre o termo que definiria essa população, sem camuflar sua condição e demostrando, com dignidade, a sua realidade. Desta forma, se chega à expressão "pessoa com deficiência" como a mais adequada, a qual traz um conceito aberto e de caráter social:

Pessoas com deficiência são aquelas que têm impedimentos de longo prazo de natureza física, mental, intelectual ou sensorial, os quais, em interação com diversas barreiras, podem obstruir sua participação plena e efetiva na sociedade em igualdades de condições com as demais pessoas (Civil, 2015).

No Brasil, a Constituição Federal de 1988 assegura o direito à saúde a todos os cidadãos, sem distinção de qualquer natureza (Brasil, 1988). No entanto, existem evidências que mostram um acesso limitado aos serviços de saúde pela população de pessoas com deficiências, seja por falta de capacitação técnica e recursos para atendimento desta população, seja por cobertura insuficiente destes indivíduos por redes de serviços especializados (Rocha; Saintrain; Vieira-Meyer, 2015; Haddad, Tagle; Passos, 2016).

Considerando a Odontologia e a potencial vulnerabilidade da pessoa com deficiência é perceptível que existem dificuldades de acesso aos serviços de saúde bucal, tais como: barreiras arquitetônicas; de comunicação entre profissionais, pacientes e cuidadores e o acesso à informação destes indivíduos (Rocha; Saintrain; Vieira-Meyer, 2015; Gerreth; BorysewiczLewicka, 2016).

Higiene bucal inadequada, cárie não tratada, gengivite e ausências dentárias são os principais agravos de saúde bucal das pessoas com deficiência, que apresentam uma alta necessidade de procedimentos odontológicos de urgência (Rojas et al., 2016; Nunes et al., 2017; Zhou; Wong; Mcgrath, 2019; Vetorazzo et al., 2020), estando esse panorama associado a um impacto negativo no que se refere à qualidade de vida de tais indivíduos (El-Meligy et al., 2016). Também se enfatiza que procedimentos odontológicos nesses pacientes requerem mais tempo, envolvimento e cooperação mútua (Gerreth; BorysewiczLewicka, 2016).

$\mathrm{O}$ atendimento odontológico às pessoas com deficiência difere daquele instituído aos demais. Inicialmente, são mais demorados por necessitar de um condicionamento adequado às diversas demandas existentes. Nesse contexto, existe o papel fundamental do profissional que irá conduzir o caso, fazendo a abordagem tanto do paciente, como também dos cuidadores e/ou sua família, quanto às condições bucais, planejamento odontológico e execução. Por outro lado, além de existirem fragilidades no próprio atendimento desses pacientes, ainda ocorre a possibilidade de haverem barreiras e dificuldades no 
acesso aos serviços de saúde bucal enfrentado pelos pais e/ou cuidadores quando necessitam. Deste modo é importante saber e quantificar tais informações no intuito de promover políticas públicas de adequação à qualidade de serviço prestado para esse público tão importante em nossa sociedade, que requerem uma atenção e cuidado especial, lhes garantindo uma melhor qualidade de vida e diminuição das desigualdades sociais.

Portanto, o objetivo desse estudo foi identificar barreiras quanto ao acesso aos serviços de saúde bucal e as dificuldades de busca e obtenção de atendimento odontológico de crianças e adolescentes com deficiência, institucionalizados, usuários da Associação de Pais e Amigos de Excepcionais, de Campina Grande, PB (APAE-CG).

\section{Metodologia}

Tratou-se de uma pesquisa observacional, descritiva, quantitativa, do tipo transversal (Antunes; Peres, 2006), cujo Projeto foi aprovado pelo Comitê de Ética em Pesquisa da Universidade Estadual da Paraíba (Parecer $\mathrm{n}^{\circ}$ 35509714.4.0000.5187).

Durante o período de coleta de dados, o Universo desse estudo foi constituído por 103 cuidadores/responsáveis de crianças e adolescentes com deficiência, usuários da APAE-CG. Propôs-se então, a realização de um censo. Todavia, ocorreram 7 recusas por parte destes cuidadores/responsáveis, que foram contabilizadas como perda amostral. Sendo assim, a amostra constituiu-se por 96 participantes.

Entre os critérios de inclusão destacam-se: cuidadores/responsáveis por crianças ou adolescentes com deficiência, entre 0 e 19 anos de idade, cadastradas na APAE-CG, no Estado da Paraíba, que estivessem acompanhando os menores durante a coleta de dados. Os critérios de exclusão considerados foram: cuidadores/responsáveis de usuários não cadastrados na APAE-CG, ou que estes estivessem fora da faixa etária proposta.

A coleta de dados foi realizada através de entrevista junto aos cuidadores/responsáveis, com formulário adaptado do estudo de Aragão et al. (2011). A fidedignidade das respostas foi testada pelo método de validação de face com $10 \%$ dos participantes, onde durante a explicação inicial da finalidade da pesquisa e primeiro contato dos sujeitos da pesquisa com os questionários foi solicitado que estes explicassem, com suas próprias palavras, o que haviam entendido sobre cada pergunta (Frankfort-Nachmias; Nachmias, 1996). Nesse instrumento foram contemplados dados sobre as condições sociodemográficas da família, a saúde bucal da criança ou adolescente e questões relacionadas ao acesso de serviço de saúde bucal por este grupo de menores.

Ao tratar-se do acesso ao serviço, o formulário contemplou questões quanto à procura por algum serviço de saúde bucal para o menor pelo cuidador, o local de procura e se o atendimento foi prestado neste. Também se questionou as dificuldades quanto ao manejo do paciente ou capacitação do profissional, o motivo de não procurar o serviço ou de não o conseguir e a satisfação com o serviço odontológico prestado.

Os cuidadores/responsáveis foram caracterizados quanto ao grau de parentesco, escolaridade e exercício de atividade laboral, enquanto os menores foram categorizados quanto ao sexo, escolaridade, município de residência. Aplicaram-se, ainda, medidas de tendência central para as variáveis idade e renda familiar.

A análise dos dados foi feita através de técnicas de estatística descritiva por distribuições absolutas e percentuais através do software IBM SPSS Statistics versão 20.0.

\section{Resultados}

Ao término da coleta de dados, responderam ao questionário 96 cuidadores/responsáveis de crianças e adolescentes. Um total de 7 cuidadores recusaram-se a participar da investigação, representando uma taxa de não resposta de 6,7\%. A maioria das responsáveis foi representada por mães, com mais de 8 anos de estudo e que não desempenhavam nenhuma 
atividade laboral (Tabela 1).

Tabela 1. Caracterização dos cuidadores/responsáveis quanto ao parentesco, à escolaridade e à ocupação. Campina Grande, PB, 2020.

\begin{tabular}{|c|c|c|}
\hline Parentesco & $\mathbf{n}$ & $\%$ \\
\hline Mãe & 85 & 88,5 \\
\hline Pai & 2 & 2,1 \\
\hline Avô/Avó & 5 & 5,2 \\
\hline Irmã & 1 & 1,0 \\
\hline Tio/Tia & 3 & 3,1 \\
\hline Total & 96 & 100,0 \\
\hline Escolaridade & $\mathbf{n}$ & $\%$ \\
\hline$<=8$ anos & 46 & 47,9 \\
\hline$>8$ anos & 50 & 52,1 \\
\hline Total & 96 & 100,0 \\
\hline Exerce atividade remunerada & $\mathbf{n}$ & $\%$ \\
\hline Sim & 23 & 23,9 \\
\hline Não & 73 & 76,1 \\
\hline Total & 96 & 100,0 \\
\hline
\end{tabular}

Fonte: Autores.

Quanto às crianças e adolescentes pesquisados, a maioria pertencia ao sexo masculino, não frequentavam a escola e o tipo de deficiência predominante foi a Paralisia Cerebral. A maioria residia em municípios circunvizinhos à Campina Grande, com idade média de 8,26 anos $( \pm 4,99)$ e renda familiar de aproximadamente $2,15( \pm 0,52)$ salários mínimos, a época da pesquisa (Tabela 2). 
Tabela 2. Caracterização das crianças e adolescentes quanto ao sexo, escolaridade, tipo de deficiência, município de residência e segundo as medidas de tendência central para as variáveis idade e renda familiar. Campina Grande, PB, 2020.

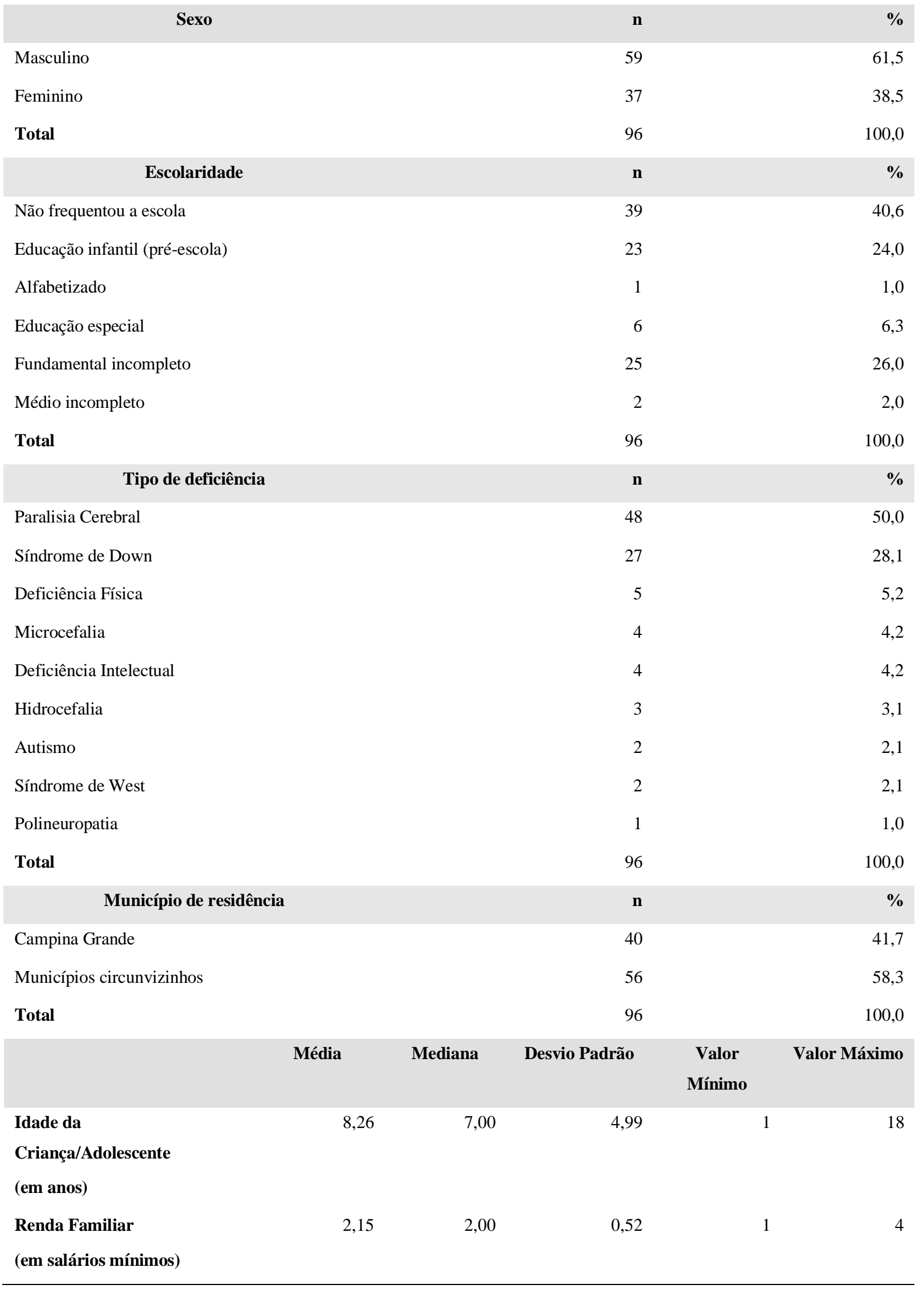


Mais da metade dos cuidadores já havia levado a criança/adolescente ao Dentista, e 51,6\% desses, há menos de 6 meses. Destaca-se que o serviço mais buscado foi a ESF (Estratégia Saúde da Família). De maneira geral, a maioria dos usuários relatou ter conseguido atendimento, sendo as recusas encontradas em maior número quando o serviço buscado foi a ESF; o que não foi retratado quando o serviço buscado foi o CEO (Centro de Especialidades Odontológicas), o setor privado e o HU (Hospital Universitário) (Tabela 3).

Tabela 3. Avaliação do acesso e acessibilidade aos serviços de saúde bucal pelas crianças e adolescentes. Campina Grande, $\mathrm{PB}, 2020$.

\begin{tabular}{|c|c|c|}
\hline Já foi ao Dentista & $\mathbf{n}$ & $\%$ \\
\hline Sim & 62 & 64,6 \\
\hline Não & 34 & 35,4 \\
\hline Total & 96 & 100,0 \\
\hline Última vez que procurou & $\mathbf{n}$ & $\%$ \\
\hline$<6$ meses & 32 & 51,6 \\
\hline De 6 meses a 1 ano & 11 & 17,7 \\
\hline De 1 a 2 anos & 17 & 27,4 \\
\hline Mais de 2 anos & 2 & 3,2 \\
\hline Total & 62 & 100,0 \\
\hline Local que procurou atendimento & $\mathbf{n}$ & $\%$ \\
\hline ESF & 19 & 30,6 \\
\hline ESF e CEO & 13 & 21,0 \\
\hline Privado & 13 & 21,0 \\
\hline $\mathrm{CEO}$ & 7 & 11,3 \\
\hline $\mathrm{HU}$ & 6 & 9,7 \\
\hline ESF e Privado & 3 & 4,8 \\
\hline ESF, CEO e Privado & 1 & 1,6 \\
\hline Total & 62 & 100,0 \\
\hline Conseguiu atendimento & n & $\%$ \\
\hline Sim & 53 & 85,5 \\
\hline Não & 9 & 14,5 \\
\hline Total & 62 & 100,0 \\
\hline Conseguiu atendimento na ESF & $\mathbf{n}$ & $\%$ \\
\hline Sim & 14 & 38,9 \\
\hline Não & 22 & 61,1 \\
\hline Total & 36 & 100,0 \\
\hline Conseguiu atendimento no CEO & $\mathbf{n}$ & $\%$ \\
\hline Sim & 19 & 90,5 \\
\hline Não & 2 & 9,5 \\
\hline Total & 21 & 100,0 \\
\hline $\begin{array}{c}\text { Conseguiu atendimento no setor } \\
\text { privado }\end{array}$ & $\mathbf{n}$ & $\%$ \\
\hline Sim & 16 & 94,1 \\
\hline Não & 1 & 5,9 \\
\hline Total & 17 & 100,0 \\
\hline
\end{tabular}




\begin{tabular}{lrr}
\multicolumn{1}{c}{ Conseguiu atendimento no HU } & $\mathbf{n}$ & $\boldsymbol{\%}$ \\
Sim & 5 & 83,3 \\
Não & 1 & 16,7 \\
Total & 6 & 100,0 \\
\hline
\end{tabular}

Fonte: Autores.

Quanto às dificuldades durante o atendimento/procedimento, a maioria afirmou não tê-las encontrado e mostraram satisfação com o serviço oferecido. Entre os que relataram ter dificuldade, a queixa principal referiu-se à recusa do profissional da ESF em atender pacientes com deficiência. Dos que não conseguiram atendimento, o motivo principal relatado foi a ausência de preparo profissional para lidar com os pacientes (Tabela 4).

Tabela 4. Avaliação das dificuldades relatadas pelos responsáveis na procura e obtenção do atendimento odontológico. Campina Grande, PB, 2020.

\begin{tabular}{|c|c|c|}
\hline $\begin{array}{l}\text { Teve dificuldade durante } o \\
\text { atendimento/procedimento }\end{array}$ & $\mathbf{n}$ & $\%$ \\
\hline Sim & 17 & 32,1 \\
\hline Não & 36 & 67,9 \\
\hline Total & 53 & 100,0 \\
\hline Tipos de dificuldades & $\mathbf{n}$ & $\%$ \\
\hline Abertura bucal do Paciente & 3 & $17,7 \%$ \\
\hline Falta de colaboração do Paciente & 3 & $17,7 \%$ \\
\hline Profissional sem capacitação & 2 & $11,8 \%$ \\
\hline Recusa do profissional da ESF em atender & 9 & $52,9 \%$ \\
\hline Total & 17 & 100,0 \\
\hline Ficou satisfeito com o atendimento & $\mathbf{n}$ & $\%$ \\
\hline Sim & 51 & 96,2 \\
\hline Não & 2 & 3,8 \\
\hline Total & 53 & 100,0 \\
\hline Motivo de não ter sido atendido & $\mathbf{n}$ & $\%$ \\
\hline Indisponibilidade de vaga & 3 & 33,3 \\
\hline $\begin{array}{l}\text { Equipamento quebrado / serviço sem } \\
\text { funcionar }\end{array}$ & 1 & 11,1 \\
\hline Despreparo profissional & 5 & 55,6 \\
\hline Total & 9 & 100,0 \\
\hline $\begin{array}{c}\text { Motivo de não ter procurado o serviço } \\
\text { odontológico }\end{array}$ & $\mathbf{n}$ & $\%$ \\
\hline Não necessitou & 27 & 79,4 \\
\hline Problemas relacionados ao serviço & 7 & 20,6 \\
\hline Total & 34 & 100,0 \\
\hline
\end{tabular}

Fonte: Autores.

\section{Discussão}

A adesão ao tratamento e a colaboração infantil no contexto odontológico, assim como os comportamentos não colaborativos e os níveis de ansiedade, que atrasam ou impedem a execução dos procedimentos, apresentam correlação 
significativa com a organização do ambiente familiar, principalmente quanto à responsividade ou permissividade das mães (Brandenburg; Marinho-Casanova, 2013). No presente estudo constatou-se, assim como em outros estudos (Gardner; Bundy; Dew, 2016; Isa et al., 2016; Lemos; Katz, 2016; Fragôso et al., 2021), que a quase totalidade dos cuidadores das crianças e adolescentes eram suas mães, o que reafirma seu papel essencial na busca do serviço odontológico. Mas também representa um risco, já que a sobrecarga em decorrência do cuidado, principalmente quando ele é de responsabilidade de uma única pessoa, está associado com problemas de saúde, sejam físicos ou emocionais (Isa et al., 2016; Lemos; Katz, 2016).

Tem-se observado na Literatura uma associação entre a escolaridade dos cuidadores e a situação de saúde bucal dos indivíduos com deficiência, seja pela maior procura por serviços entre os cuidadores com maior escolaridade (Chen et al., 2016) ou pela condição de saúde deficiente quando os cuidadores apresentam baixa escolaridade (Diab et al., 2017; Oliveira et al., 2018). Neste estudo a maioria dos cuidadores/responsáveis apresentou escolaridade acima de oito anos. No entanto, não deixa de ser um dado preocupante ao associarmos com os demais fatores de risco a qual a população estudada está associada.

No presente estudo, as famílias apresentavam uma renda familiar de dois salários mínimos. Dado que, se refletido considerando os achados de Isa et al. (2016) e de Correia e Salimene (2016), torna-se preocupante; visto que, as famílias de crianças com deficiência enfrentam maiores encargos financeiros do que as famílias que têm filhos não-deficientes. Estes autores ainda destacaram que a maioria dessas famílias recebe algum auxílio assistencial, sendo a principal fonte de renda da casa e ainda apontaram para a dificuldade ao qual os cuidadores sofrem para se manterem empregado(a)s. O presente estudo corrobora com o de Lemos e Katz (2016), já que a maioria dos cuidadores entrevistados não exercem qualquer atividade laboral.

O perfil da população de crianças e adolescentes investigados, caracterizou-se, principalmente, por ser do sexo masculino e não frequentar a escola regular. Neste contexto, a Literatura (Faker et al., 2016; Lemos; Katz, 2016; Castilho et., 2017) concorda com o presente estudo quanto a diferença em relação ao sexo. Sendo o sexo masculino mais frequente nos estudos de crianças e adolescentes com deficiência. Quanto ao acesso à educação, Lindly, Sinche e Zuckerman (2015) constataram que crianças e adolescentes entre 6 e 17 anos de idade e com deficiência, nos Estados Unidos, encontram falhas tanto na facilidade de acesso, quanto no recebimento de serviços educacionais, principalmente quando o usuário apresentava alguma limitação física. Também verificaram, assim como esse estudo, que metade desse grupo não frequentava escolas.

Mais da metade dos entrevistados vinham de cidades circunvizinhas para a APAE, em Campina Grande-PB, o que, para pacientes com limitações, pode representar um aumento nos desafios de acesso aos serviços de saúde: maior distância dos serviços, custos de transporte e estradas ruins, falta de transporte público ou de adaptações para esses usuários e, para os que dependem de carona, perda do senso de liberdade/autonomia para utilizar os serviços (Mji et al., 2017).

A média de idade dos pacientes deste estudo foi abaixo da primeira década de vida, quando os problemas em saúde bucal tendem a ser solucionados mais facilmente e as atividades em Educação e Promoção de Saúde são mais eficazes (Domingues et al., 2015). Esse dado, apesar de positivo, associado às dificuldades de acesso constatadas, a falta de colaboração e as dificuldades inerentes de cada deficiência podem levar esses pacientes, em idades mais avançadas, a ter tratamentos curativos mais extensos e/ou mutiladores.

Quanto à busca por serviços de saúde bucal, mais de um terço dos cuidadores entrevistados nunca levaram as crianças e adolescentes ao Dentista e esse número parece ser uma constante entre os costumes da população brasileira. Segundo dados da última Pesquisa Nacional de Saúde (IBGE, 2015), verificou-se que na região Nordeste um pouco mais de um terço da população consultaram o Dentista nos últimos 12 meses. Sobre a população com deficiência, Rocha, Saintrain e Vieira-Meyer (2015) constataram que as pessoas com deficiência ou seus cuidadores não sentem que seu atendimento odontológico é uma prioridade e que mais da metade dessa população não costuma procurar esse serviço, salvo em emergências.

Considerando a Odontologia, as Políticas Públicas no Brasil aplicadas especificamente para este grupo propõem uma 
estratégia que está em consonância com a Política Nacional de Saúde da Pessoa Portadora de Deficiência (Portaria n. 1.060/2002), recomendando-se que os serviços se organizem para ofertar atendimento prioritário na atenção primária (Brasil, 2008). Contudo, o que se constatou nesta pesquisa foi que na atenção primária (ESF); embora seja esta a mais procurada para o atendimento destes pacientes; os entrevistados relataram que poucos conseguiram atendimento quando comparado ao serviço privado e nos níveis de atenção especializados.

Quanto às barreiras de acesso de crianças com deficiência aos cuidados em saúde bucal, Gerreth e BorysewiczLewicka (2016) e Cruz, Chi e Huebner (2016) constataram que apenas um terço dos cuidadores entrevistados em seu estudo, relataram não encontrar dificuldades nos serviços de saúde; alguns entrevistados relataram relutância dos Dentistas em atender esses pacientes, principalmente nos grupos de crianças com deficiência grave/profunda e moderada. Além disso, os dados do questionário revelaram que poucos profissionais faziam uma consulta para planejar a próxima visita, portanto o tratamento era interrompido.

Constatou-se no presente estudo que entre as dificuldades de atendimento relatadas, a recusa do profissional da ESF em atender pacientes com deficiência foi a principal queixa e, entre aqueles que não conseguiram atendimento, a falta de preparo profissional para lidar com os pacientes. Tal fato pode ser justificado pelo percentual significativo das faculdades de Odontologia do Brasil em que não havia Componente Curricular voltado ao atendimento de pacientes com deficiência, aspecto que tem gerado uma lacuna na formação dos Cirurgiões-Dentistas até então (Haddad; Tagle; Passos, 2016), e que pode estar associado aos achados desta pesquisa, no que concerne à recusa dos profissionais quando do atendimento desses pacientes; fato verificado também nos estudos de Rocha, Saintrain e Vieira-Meyer (2015). É importante ressaltar, no entanto, que um estudo recente (Cipriano et al., 2020) envolvendo apenas as Instituições Públicas de Ensino Superior demonstrou uma melhora nesse panorama. Dos 57 cursos analisados, 37 apresentam em sua grade curricular alguma disciplina sobre Pacientes com Necessidades Especiais, de caráter obrigatório ou optativo

Um número considerável de cuidadores nunca levou a criança ou o adolescente ao Dentista, sendo o principal motivo por acreditarem que os mesmos não necessitavam. No entanto, sabe-se que uma perfeita saúde bucal é atingida por meio de uma associação de comportamentos, incluindo visitas regulares preventivas ao Dentista (Cruz; Chi; Huebner, 2016). Um estudo realizado por Oliveira et al. (2016) em pacientes com paralisia cerebral destacou que a higienização da cavidade oral desses indivíduos é bastante dificultada, devido à condição do paciente, sendo que os cuidadores, por vezes disseram não conseguir realizar corretamente a higienização, nem mesmo uma vez durante o dia. Esses dados, além de reforçar a importância do cuidador na manutenção da saúde bucal desses indivíduos, levanta um alerta para a necessidade de sensibilização destes responsáveis, já que as condições de saúde e higiene oral destes indivíduos são, muitas vezes, inadequadas, como concluíram também Pini, Fröhlich e Rigo (2016) ao estudar usuários da APAE, de Passo Fundo, RS.

O acesso aos serviços de saúde bucal por pessoas com deficiência, apesar de garantido por lei, ainda é limitado. Lacunas na formação profissional dos Cirurgiões-Dentistas, falta de conhecimento e cooperação dos cuidadores e dificuldades inerentes de cada deficiência, podem reduzir ou atrasar o tratamento precoce e agravar a situação de saúde desses indivíduos. Reconhecer essas barreiras limitantes na infância e adolescência é o ponto inicial para a construção de políticas públicas para amenizar esse quadro e garantir igualdade de condições com as demais pessoas.

\section{Conclusão}

O presente estudo pôde concluir que, na amostra estudada, mais da metade das crianças e adolescentes, usuários da APAE-CG, já havia sido levada ao Dentista, principalmente há menos de 6 meses. O serviço mais buscado foi a ESF. Quando se considerou outros serviços, além da ESF, a maioria dos usuários relatou ter conseguido atendimento; no entanto, a maior parte daqueles que procuraram a ESF, afirmaram que não receberam atendimento. 
Quanto às dificuldades durante o atendimento, a maioria dos cuidadores dos usuários da APAE-CG afirmou não tê-las encontrado e mostrou satisfação com o serviço oferecido. Entre os que relataram ter dificuldade, a queixa principal referiu-se à recusa do profissional do ESF em atender pacientes com deficiência.

O presente trabalho traça um breve panorama da questão do acesso da pessoa com deficiência, em especial crianças e adolescentes ao serviço odontológico. É importante ressaltar que este tipo de estudo possibilita a elaboração de políticas públicas de saúde mais efetivas, uma vez que oferece uma análise das principais dificuldades encontradas por essa população. Sugere-se que, em estudos posteriores, haja uma maior abrangência no que diz respeito à amostra da população estudada. Além da APAE, outras instituições poderiam ser incluídas para uma análise mais abrangente, tais como: Associação de Assistência à Criança Deficiente (AACD), Associação de Amigos do Autista (AMA), Centros de Atenção Psicossocial Infanto-juvenil (CapsI) e até mesmo a Estratégia de Saúde da Família (ESF), uma vez que nem todas as crianças e adolescentes com deficiência são acompanhados por serviços especializados.

\section{Referências}

Antunes, J. L. F., Peres, M. A., \& Crivello Júnior, O. (2006). Epidemiologia da saúde bucal. In Epidemiologia da saúde bucal (pp. 441-441).

Aragão, A. K. R., Sousa, A., Silva, K., Vieira, S., \& Colares, V. (2011). Acessibilidade da criança e do adolescente com deficiência na atenção básica de saúde bucal no serviço público: estudo piloto. Pesquisa Brasileira em Odontopediatria e Clínica Integrada, 11(2), $159-164$.

Brandenburg, O. J., \& Marinho-Casanova, M. L. (2013). A relação mãe-criança durante o atendimento odontológico: contribuições da análise do comportamento. Estudos de Psicologia (Campinas), 30(4), 629-640.

Brasil, C. (2009). Decreto $n^{\circ}$ 6.949, de 25 de agosto de 2009. Promulga a Convenção Internacional sobre os Direitos das Pessoas com Deficiência e seu Protocolo Facultativo, assinados em Nova York, em 30 de março de 2007. Diário Oficial da União, (163).

Brasil, S. F. (1988). Constituição da república federativa do Brasil. Senado Federal, Centro Gráfico.

Câmara, D. D. (2009). Legislação brasileira sobre pessoas portadoras de deficiência.

Castilho, L. S., Abreu, M. H. N. G., Ribeiro, L. V. L., Souza, M. E., \& Resende, V. L. S. (2017). Perfil dos pacientes com deficiências de desenvolvimento sob atendimento odontológico em um projeto de extensão intersetorial. Arquivos em Odontologia, 53.

Chen, M. C., Kung, P. T., Su, H. P., Yen, S. M., Chiu, L. T., \& Tsai, W. C. (2016). Utilization of tooth filling services by people with disabilities in Taiwan. International journal for equity in health, 15(1), 1-10.

Civil, C. (2015). Lei No 13.146, de 6 de julho 2015. Institui a lei brasileira de inclusão da pessoa com deficiência (estatuto da pessoa com deficiência). Brasília.

Cipriano, O. B., Rolim, A. K. A., Alves, M. A. S. G., de Oliveira Filho, A. A., Guênes, G. M. T., da Costa Figueiredo, C. H. M., \& da Penha, E. S. (2020). Odontologia para pacientes especiais: análise da composição curricular das instituições públicas do Brasil. Research, Society and Development, 9(2), e189922135-e189922135.

Correia, V. D., \& de Melo Salimene, A. C. (2016). Perfil social de crianças e jovens com paralisia cerebral em um centro de reabilitação física. CEP, 4116, 040 .

Cruz, S., Chi, D. L., \& Huebner, C. E. (2016). Oral health services within community-based organizations for young children with special health care needs. Special Care in Dentistry, 36(5), 243-253.

Diab, H. A., Salameh, Z., Hamadeh, G. N., Younes, G., \& Ayoub, F. (2017). Oral health status of institutionalized individuals with intellectual disabilities in Lebanon. Journal of oral \& maxillofacial research, 8(1).

Domingues, N. B., Ayres, K. C. M., Mariusso, M. R., Zuanon, Â. C. C., \& Giro, E. M. A. (2015). Caracterização dos pacientes e procedimentos executados no serviço de atendimento a pacientes com necessidades especiais da Faculdade de Odontologia de Araraquara-UNESP. Revista de Odontologia da UNESP, $44(6), 345-350$.

El-Meligy, O., Maashi, M., Al-Mushayt, A., Al-Nowaiser, A., \& Al-Mubark, S. (2016). The effect of full-mouth rehabilitation on oral health-related quality of life for children with special health care needs. Journal of Clinical Pediatric Dentistry, 40(1), 53-61.

Faker, K., Di Lanaro, N., Paula, V. A. C., \& Tostes, M. A. (2016). Profile of special needs patients assisted in a dental project. Brazilian Dental Science, 19(4), $42-48$.

Fragôso, D. N., Silva, G. M. L. N., de Oliveira, J. J. M., Farias, M. F., da Silva Andrade, K., de Figueiredo, N. F. D., \& Cardoso, A. M. R. (2021). Utilização dos serviços odontológicos por pacientes pediátricos com Síndrome de Down de acordo com os cuidadores. Research, Society and Development, 10(4), e9010413767-e9010413767.

Gardner, K., Bundy, A., \& Dew, A. (2016). Perspectives of rural carers on benefits and barriers of receiving occupational therapy via information and communication technologies. Australian occupational therapy journal, 63(2), 117-122. 
Gerreth, K., \& Borysewicz-Lewicka, M. (2016). Access barriers to dental health care in children with disability. A questionnaire study of parents. Journal of Applied Research in Intellectual Disabilities, 29(2), 139-145.

Haddad, A. S., Tagle, E. L., \& Passos, V. D. A. B. (2016). Momento atual da Odontologia para pessoas com deficiência na América Latina: situação do Chile e Brasil. Revista da Associacao Paulista de Cirurgioes Dentistas, 70(2), 132-140.

Instituto Brasileiro de Geografia e Estatística (2015). Pesquisa nacional de saúde 2013: acesso e utilização dos serviços de saúde, acidentes e violências: Brasil, grandes regiões e unidades da federação.

Isa, S. N. I., Ishak, I., Ab Rahman, A., Saat, N. Z. M., Din, N. C., Lubis, S. H., \& Ismail, M. F. M. (2016). Health and quality of life among the caregivers of children with disabilities: A review of literature. Asian Journal of Psychiatry, 23, 71-77.

Lemos, A. C. O., \& Katz, C. R. T. (2016). Cárie dentária em crianças com paralisia cerebral e sua relação com a sobrecarga dos cuidadores. Arquivos em Odontologia, 52(2).

Lindly, O. J., Sinche, B. K., \& Zuckerman, K. E. (2015). Variation in educational services receipt among US children with developmental conditions. Academic pediatrics, 15(5), 534-543.

Miji, G., Braathen, S. H., Vergunst, R., Scheffler, E., Kritzinger, J., \& Mannan, H. Exploring the interaction of activity limitations with context, systems, community and personal factors in accessing public health care services: A presentation of South African case studies. Afr. j. prim. health care fam. med.(Online); 9 (1): a1166.

Nachmias, D., \& Nachmias, C. (1976). Research methods in the social sciences.

Nunes, R., Simões, P. W., Pires, P. D. S., \& Rosso, M. L. P. (2017). Prevalência de alterações bucais em pessoas com deficiência na clínica da universidade do extremo sul catarinense. Revista de Odontologia da Universidade Cidade de São Paulo, 29(2), 118-128.

Oliveira, E. T., Carvalho, A. C., Matinelli, J., Thedei-Júnior, G., Lepri, C. P., \& Menezes-Oliveira, M. A. H. (2016). Avaliação da condição bucal de pacientes portadores de paralisia cerebral na cidade de Uberaba. Arquivos em Odontologia, 52(2).

Oliveira, P. A. D., Bendo, C. B., Paiva, S. M., de Abreu, M. H. N. G., \& Auad, S. M. (2018). Associação do nível de escolaridade de pais e responsáveis com os hábitos alimentares e de higiene bucal de crianças. Arquivos em Odontologia, 54.

Pini, D. D. M., Fröhlich, P. C. G. R., \& Rigo, L. (2016). Avaliação da saúde bucal em pessoas com necessidades especiais. Einstein, 14(4), $501-507$.

Rocha, L. L., de Lima Saintrain, M. V., \& Vieira-Meyer, A. P. G. F. (2015). Access to dental public services by disabled persons. BMC oral health, 15(1), 1-9.

Rojas, C. F., Wichrowska-Rymarek, K., Pavlic, A., Vinereanu, A., Fabjanska, K., Kaschke, I., \& Marks, L. A. (2016). Oral health needs of athletes with intellectual disability in Eastern Europe: Poland, Romania and Slovenia. International dental journal, 66(2), 113-119.

Vetorazzo, K. R. S., Rolim, T. D. F. A., Rolim, A. K. A., Guedes, M. C. B. M., \& de Souza, S. L. X. (2020). Prevalência de alterações bucais em pacientes com necessidades especiais. Research, Society and Development, 9(2), e146922148-e146922148.

Zhou, N., Wong, H. M., \& McGrath, C. (2019). Oral health and associated factors among preschool children with special healthc are needs. Oral diseases, $25(4), 1221-1228$ 